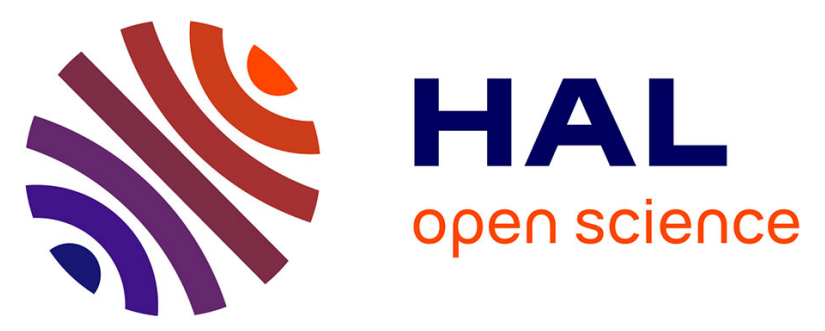

\title{
Palladium and platinum complexes with planar chiral 1,2-disubstituted ferrocenes containing phosphine and thioether donor groups
}

Raluca Malacea, Lucie Routaboul, Eric Manoury, Jean-Claude Daran, Rinaldo Poli

\section{To cite this version:}

Raluca Malacea, Lucie Routaboul, Eric Manoury, Jean-Claude Daran, Rinaldo Poli. Palladium and platinum complexes with planar chiral 1,2-disubstituted ferrocenes containing phosphine and thioether donor groups. Journal of Organometallic Chemistry, 2008, 693 (8-9), pp.1469-1477. 10.1016/j.jorganchem.2007.08.021 . hal-03192714

\section{HAL Id: hal-03192714 \\ https://hal.science/hal-03192714}

Submitted on 8 Apr 2021

HAL is a multi-disciplinary open access archive for the deposit and dissemination of scientific research documents, whether they are published or not. The documents may come from teaching and research institutions in France or abroad, or from public or private research centers.
L'archive ouverte pluridisciplinaire HAL, est destinée au dépôt et à la diffusion de documents scientifiques de niveau recherche, publiés ou non, émanant des établissements d'enseignement et de recherche français ou étrangers, des laboratoires publics ou privés. 


\section{Palladium and Platinum Complexes with Planar Chiral 1,2-disubstituted ferrocenes containing phosphine and thioether donor groups ${ }^{\dagger}$}

Raluca Malacea, Lucie Routaboul, Eric Manoury, * Jean-Claude Daran and Rinaldo Poli*

Laboratoire de Chimie de Coordination, UPR CNRS 8241 liée par convention à l'Université

Paul Sabatier et à l'Institut National Polytechnique de Toulouse, 205 Route de Narbonne,

31077 Toulouse Cedex, France.

E-mails : manoury@1cc-toulouse.fr and inaldo.Poli@lcc-toulouse.fr

Proofs to:

Rinaldo Poli

Tel: $+33-561333173$

Fax: +33-561553003

E-mail: Rinaldo.Poli@Icc-toulouse.fr

${ }^{\dagger}$ Dedicated to the memory of F. Albert Cotton, a caring mentor and a good friend 


\section{Summary}

Three palladium(II) complexes and four platinum(II) complexes having general formula $\mathrm{CpFe}\left\{1,2-\mathrm{C}_{5} \mathrm{H}_{3}\left(\mathrm{PPh}_{2}\right)\left(\mathrm{CH}_{2} \mathrm{SR}\right)\right\} \mathrm{MCl}_{2}(\mathrm{M}=\mathrm{Pd}, \mathrm{R}=\mathrm{Ph}$, Et and $t \mathrm{Bu} ; \mathrm{M}=\mathrm{Pt}, \mathrm{R}=\mathrm{Ph}$, Et, $t \mathrm{Bu}$ and $\mathrm{Cy}$ ) have been synthesized by reaction of the corresponding $\mathrm{CpFe}\{1,2-$ $\left.\mathrm{C}_{5} \mathrm{H}_{3}\left(\mathrm{PPh}_{2}\right)\left(\mathrm{CH}_{2} \mathrm{SR}\right)\right\}$ ligands with $\mathrm{PdCl}_{2}\left(\mathrm{CH}_{3} \mathrm{CN}\right)_{2}$ or $\mathrm{PtCl}_{2}\left(\mathrm{CH}_{3} \mathrm{CN}\right)_{2}$. These complexes have been fully characterized in solution and in solid state. In all cases, monomeric square planar complexes were obtained as pure diastereoisomers.

\section{Keywords}

Palladium, Platinum, ferrocene chemistry, planar chirality, phosphine ligands, thioether ligands. 


\section{Introduction}

Since its discovery and early studies, which actively involved F. A. Cotton [1, 2], ferrocene has come a long way as a tool in chemistry, comprising applications in electrochemistry [3], material science [4-6], synthesis, and catalysis, particularly enantioselective catalysis by way of the planar chirality [7] associated to an asymmetric 1,2 disubstitution [8]. Important examples (see Scheme 1) are P,P ligands like TRAP [9-13], Josiphos [14], and particularly the industrially important Xyliphos [15], Taniaphos [16-18] and Walphos-type [19] ligands, (P,N) ligands, especially phosphine-oxazolines like, for instance DIPOF [20], and more recently (P,S) ligands like Fesulphos [21].

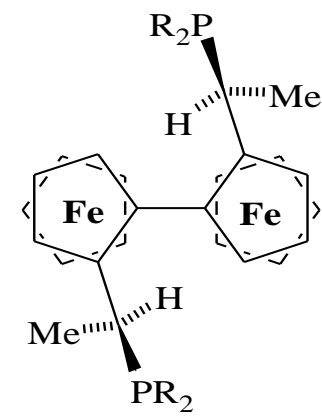

TRAP

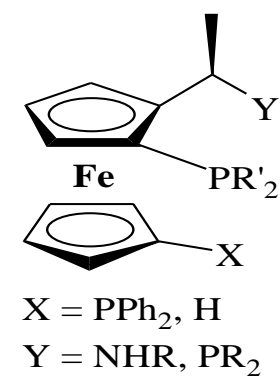

Josiphos

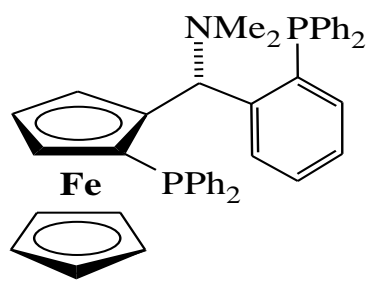

Taniaphos

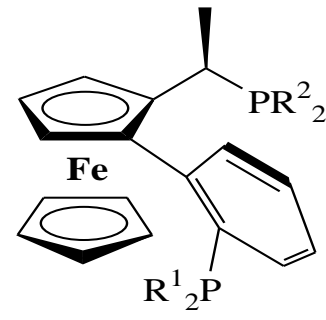

Walphos

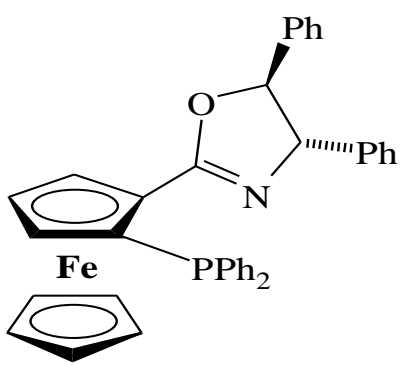

DIPOF

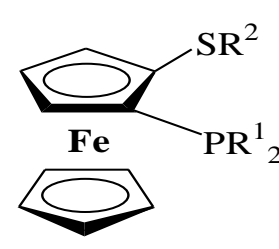

Fesulphos

\section{Scheme 1}

We have recently developed new chiral ferrocenyl (P,S) ligands with general formula $\mathrm{CpFe}\left\{1,2-\mathrm{C}_{5} \mathrm{H}_{3}\left(\mathrm{PPh}_{2}\right)\left(\mathrm{CH}_{2} \mathrm{SR}\right)\right\}$ [22], characterized by having only planar chirality, in both 
racemic and enantiomerically pure forms ( $\mathrm{R}$ or $\mathrm{S}$ configuration) [23] and briefly reported on their coordination chemistry $[24,25]$. These ligands could be successfully applied to a few asymmetric catalytic reactions, namely palladium-catalyzed allylic substitution $[22,26]$ and iridium-catalyzed ketone hydrogenation [27]. In this paper we report on the synthesis and characterization of $\mathrm{Pd}^{\mathrm{II}}$ and $\mathrm{Pt}^{\mathrm{II}}$ complexes containing a variety of these $(\mathrm{P}, \mathrm{S})$ ligands. $\mathrm{A}$ useful aspect of this work is the structural verification of a systematic control of the coordinated sulphur atom chirality by the ferrocene absolute configuration, which is expected to be key in the transmission of the chiral information to the catalytic metal.

\section{Results and Discussion}

The synthetic procedures involved a ligand exchange process on the appropriate $\mathrm{MCl}_{2}(\mathrm{MeCN})_{2}$ precursor $(\mathrm{M}=\mathrm{Pd}, \mathrm{Pt})$, replacing the two $\mathrm{MeCN}$ ligands with the desired racemic $(\mathrm{P}, \mathrm{S})$ ligand, see Scheme 2. Four thioether ligands were used for this study, having phenyl, ethyl, tert-butyl and cyclohexyl as substituents on the sulphur atom. The reaction takes place in dichloromethane at room temperature for the palladium systems, whereas it requires much harsher conditions (toluene reflux) for platinum, in agreement with the greater lability of $\mathrm{Pd}^{\mathrm{II}}$ for ligand exchange processes. Dark red $(\mathrm{Pd})$ and orange $(\mathrm{Pt})$ solids were obtained in good to excellent yields.

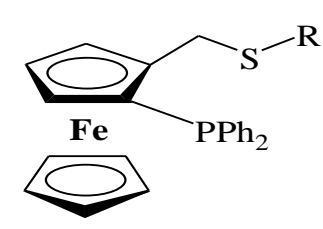

1

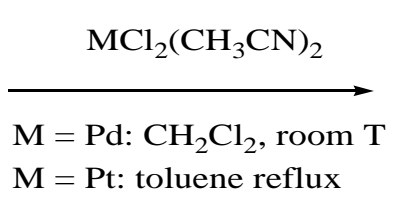

$\mathrm{M}=\mathrm{Pt}$ : toluene reflux

$$
\mathbf{R}=\mathbf{P h}(\mathbf{a}), \mathbf{E t}(\mathbf{b}), t \mathbf{B u}(\mathbf{c}), \mathrm{Cy}(\mathrm{d})
$$

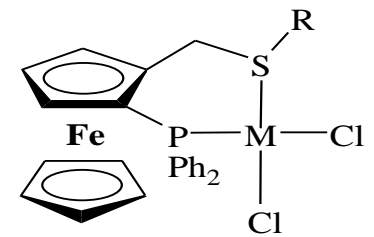

$2(M=P d)$

$3(\mathbf{M}=\mathbf{P t})$

Scheme 2. 
All reactions were carried out with the recemic form of the ligands, thus leading to the products as racemic mixtures. However, the coordination of the thioether function generates a new chiral center on the sulphur atom, thus two distinct diasteroisomers may be obtained in principle. In fact, the reactions are $100 \%$ diastereoselective, leading in each case to only one observable product, as shown by NMR spectroscopy. Each product gives one single signal in the ${ }^{31} \mathrm{P}$ NMR spectrum and one set of resonances, notably only one signal for the unsubstituted $\mathrm{Cp}$ ring, in the ${ }^{1} \mathrm{H}$ NMR spectrum. In addition, complexes $\mathbf{3}$ show the expected satellites corresponding to the Pt-P coupling for the ${ }^{31} \mathrm{P}$ NMR signal. The ${ }^{31} \mathrm{P}$ and ${ }^{195} \mathrm{Pt} \mathrm{NMR}$ data are shown in Table 1. The three complexes 2 have analogous ${ }^{31} \mathrm{P}$ chemical shifts (see Table 1) indicating similar structures in solution. In the same way, the four complexes $\mathbf{3}$ have analogous ${ }^{31} \mathrm{P}$ and ${ }^{195} \mathrm{Pt}$ chemical shifts (see Table 1), leading to the same conclusion. The ${ }^{195} \mathrm{Pt}$ chemical shifts of compounds 3 (from $-4030 \mathrm{ppm}$ to $-4095 \mathrm{ppm}$ ) are in the expected range for such cis-Pt(II) complexes and approximately midway relative to complexes containing two $\mathrm{P}$ ligands (cis- $\mathrm{PtCl}_{2}\left(\mathrm{PMe}_{3}\right)_{2}$ : $\left.-4408 \mathrm{ppm}\right)$ and two $\mathrm{S}$ ligands $\left(\mathrm{cis}-\mathrm{PtCl}_{2}\left(\mathrm{SMe}_{2}\right)_{2}\right.$ : -3551 ppm) [28]. The Pt-P coupling constant agrees with previously reported values for cissquare planar $\mathrm{PtCl}_{2} \mathrm{LL}$ ' structures containing phosphine-thioether $\left(\mathrm{J}_{\mathrm{P}-\mathrm{Pt}}=3680 \mathrm{~Hz}\right)$ [29] or diphosphine $\left(\mathrm{J}_{\mathrm{P}-\mathrm{Pt}}=3650 \mathrm{~Hz}\right.$ [29], $3573 \mathrm{~Hz}$ [30], $3693 \mathrm{~Hz}$ [31], $3810 \mathrm{~Hz}$ [32]) ligands.

Table 1. ${ }^{31} \mathrm{P}$ and ${ }^{195} \mathrm{Pt}$ NMR data for compounds $\mathbf{2}$ and $\mathbf{3}$.

\begin{tabular}{|l|c|c|c|}
\hline & $\begin{array}{c}\mathbf{N M R}{ }^{31} \mathbf{P}, \boldsymbol{\delta}(\mathbf{p p m}) \\
\left(\mathrm{CDCl}_{3}, 500 \mathrm{MHz}\right)\end{array}$ & $\begin{array}{c}\mathbf{N M R}{ }^{195} \mathbf{P t}, \boldsymbol{\delta}(\mathbf{p p m}) \\
\left(\mathrm{CDCl}_{3} .500 \mathrm{MHz}\right)\end{array}$ & $\mathbf{J P P t} \mathbf{( H z})$ \\
\hline $\mathbf{2 a}$ & 20.9 & & \\
\hline 2b & 21.2 & & \\
\hline 3a & 22.4 & & 3608 \\
\hline 3b & -0.8 & -4093 & 3666 \\
\hline 3c & -3.68 & -4110 & 3636 \\
\hline
\end{tabular}




\begin{tabular}{|l|l|l|l|}
\hline 3d & -0.5 & -4095 & 3660 \\
\hline
\end{tabular}

Single crystals of each complex $\mathbf{2}$ and $\mathbf{3}$ were obtained by slow diffusion of hexane in dichloromethane solutions and used for structural studies by X-ray diffraction. Few other phosphine-thioether- $\mathrm{MCl}_{2}(\mathrm{M}=\mathrm{Pd}, \mathrm{Pt})$ complexes have previously been characterized by $\mathrm{X}$ ray diffraction. A few relevant examples for Pd are compounds A-I [33-37] in Scheme 3, whereas those of Pt include compounds $\mathbf{J}$ [38], K [39] and L [40], shown in Scheme 4. Views of the three Pd structures are shown in Figure 1 (2a), Figure 2 (2b), Figure 3 (2c). The structures of complexes $\mathbf{3 a}$ and $\mathbf{3 b}$ are essentially identical to those of the corresponding $\mathbf{P d}$ complexes. A view of the Pt complexes $\mathbf{3 c}$ and $\mathbf{3 d}$ are shown in Figure 3 and Figure 4, respectively.<smiles>CC1CC(Cl)[Te](Cl)(Cl)(c2ccccc2)[SH](C)C1</smiles>

A<smiles>CC[SH]1C(C)C2CC2P(Cl)(c2ccccc2)(c2ccccc2)[Te]1(Cl)c1ccccc1</smiles>

$\mathbf{F}$<smiles>ClP(c1ccccc1)[Te](Cl)(Cl)c1ccccc1</smiles>

B<smiles></smiles>

$\mathbf{C}$<smiles>Cc1cccc(C)c1[SH]1CC2C(C2(C)C)P(Cl)(c2ccccc2)(c2ccccc2)[Te]1(Cl)Cl</smiles>

$\mathbf{D}$<smiles>Cc1cccc(C)c1[SH]1C(C)C2CC2[PH](c2ccccc2)(c2ccccc2)[Te]1(Cl)Cl</smiles>

$\mathbf{E}$<smiles>C[SH]1c2ccccc2CP(c2ccccc2)[Te](Cl)(c2ccccc2)[Z]1(Cl)Cl</smiles>

G<smiles>C[Y5]1[SH](C)CCCP(c2ccccc2)[Te]1(Cl)c1ccccc1</smiles>

$\mathbf{H}$

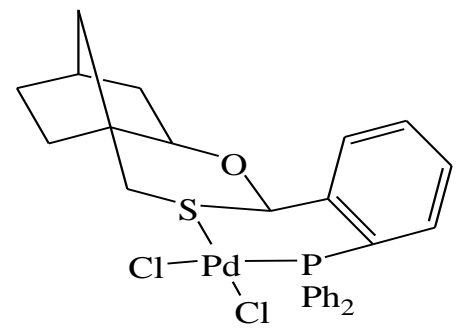

I

\section{Scheme 3.}

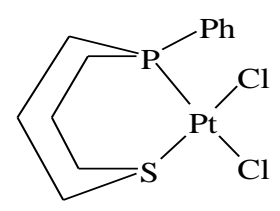

J

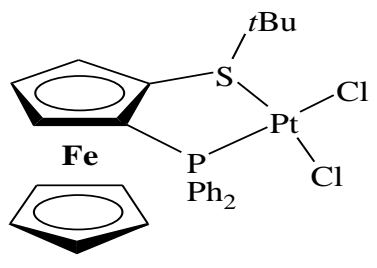

K

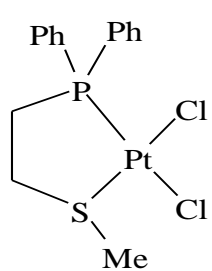

L

\section{Scheme 4.}




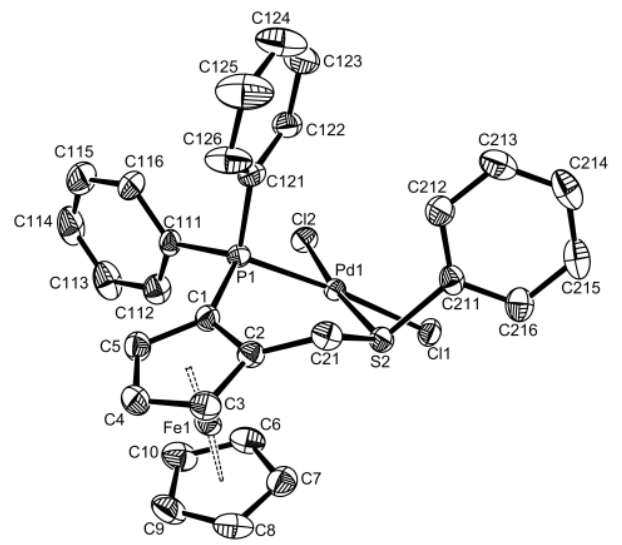

Figure 1. ORTEP view of the palladium complex 2a.

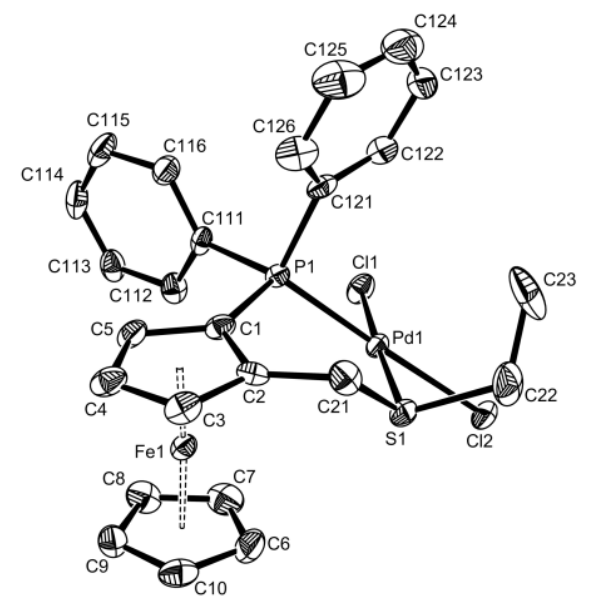

Figure 2. ORTEP view of the palladium complex $\mathbf{2 b}$.
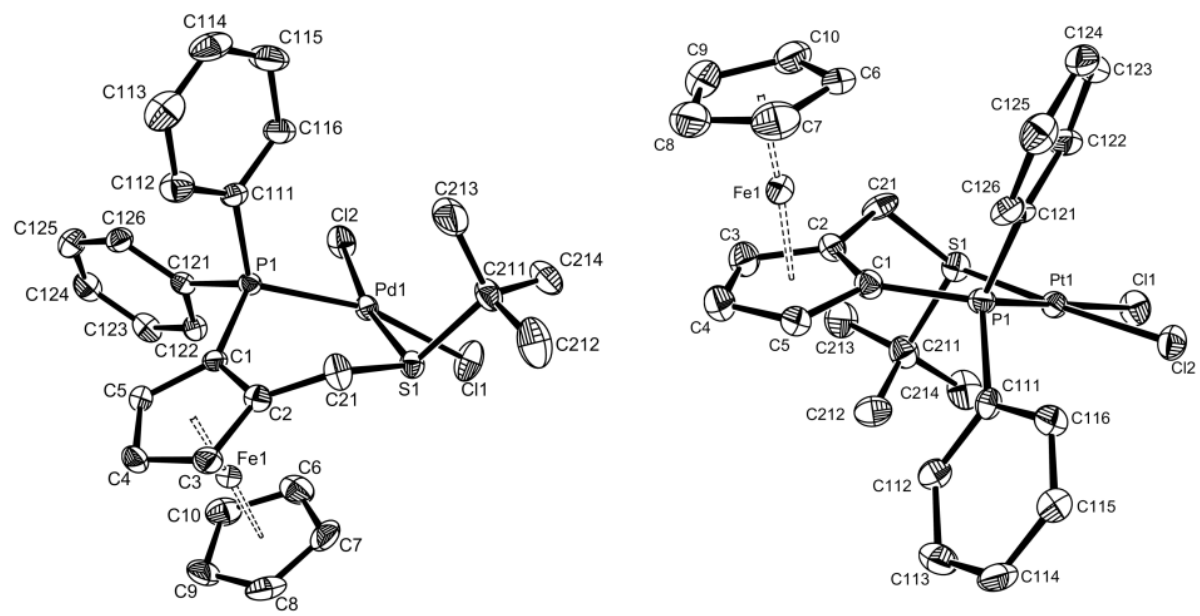

Figure 3. ORTEP view of the palladium complex $\mathbf{2 c}$ and the platinum complex $\mathbf{3 c}$. 


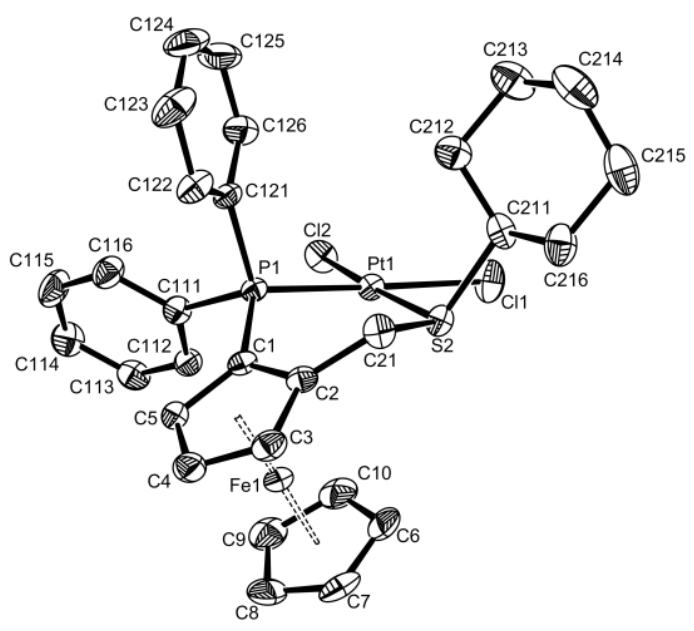

Figure 4. ORTEP view of the platinum complex 3d.

All compounds adopt a mononuclear square planar geometry with the phosphine and thioether functions in relative cis position. In each case, the sulphur substituent is placed opposite (anti) to the FeCp group, with respect to the S-C-C-C-P chelate. Thus, the metal atom has been selectively coordinated by one of the two lone pairs of the sulphur atom; after coordination, the remaining lone pair is oriented syn to the unsubstituted Cp. Consequently,

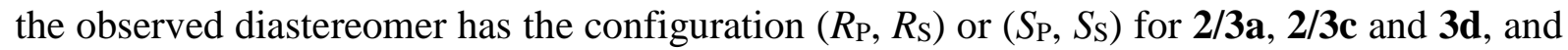
$\left(R_{\mathrm{P}}, S_{\mathrm{S}}\right)$ or $\left(S_{\mathrm{P}}, R_{\mathrm{S}}\right)$ for $\mathbf{2} / \mathbf{3 b}$. Relevant bond distances and angles for all compounds are compared in Table 2 .

Table 2. Selected bond distances $(\AA)$ and angles $\left(^{\circ}\right)$ for compounds $\mathbf{2}$ and $\mathbf{3}$.

\begin{tabular}{|l|c|c|c|c|c|c|c|}
\hline & \multicolumn{3}{|c|}{ M = Pd } & \multicolumn{4}{c|}{ M Pt } \\
\hline & 2a & 2b & 2c & 3a & 3b & 3c & 3d \\
\hline M-P & $2.2311(10)$ & $2.227(2)$ & $2.2251(8)$ & $2.2281(10)$ & $2.2184(12)$ & $2.2395(18)$ & $2.2162(8)$ \\
\hline M-S & $2.3074(9)$ & $2.308(2)$ & $2.3215(8)$ & $2.2717(11)$ & $2.2826(13)$ & $2.2718(19)$ & $2.2838(8)$ \\
\hline M-Cl $(t-S)$ & $2.3102(9)$ & $2.313(2)$ & $2.3006(8)$ & $2.3019(11)$ & $2.3173(13)$ & $2.3123(19)$ & $2.3003(8)$ \\
\hline M-Cl $(t-\mathrm{P})$ & $2.3683(10)$ & $2.365(2)$ & $2.3588(8)$ & $2.3727(11)$ & $2.3694(12)$ & $2.3534(17)$ & $2.3537(8)$ \\
\hline \multicolumn{9}{|c|}{} \\
\hline P-M-S & $95.08(4)$ & $93.90(6)$ & $96.08(3)$ & $95.56(4)$ & $94.71(5)$ & $99.44(7)$ & $95.96(3)$ \\
\hline P-M-Cl $(c)$ & $90.20(3)$ & $89.80(5)$ & $88.99(3)$ & $90.21(4)$ & $91.03(4)$ & $85.92(7)$ & $90.65(3)$ \\
\hline
\end{tabular}




\begin{tabular}{|l|c|c|c|c|c|c|c|}
\hline $\mathrm{P}-\mathrm{M}-\mathrm{Cl}(t)$ & $175.56(3)$ & $176.50(7)$ & $163.68(3)$ & $176.61(4)$ & $177.32(5)$ & $174.08(7)$ & $178.55(3)$ \\
\hline $\mathrm{S}-\mathrm{M}-\mathrm{Cl}(c)$ & $83.41(3)$ & $83.75(6)$ & $86.73(3)$ & $84.14(4)$ & $83.71(5)$ & $86.23(7)$ & $84.84(3)$ \\
\hline $\mathrm{S}-\mathrm{M}-\mathrm{Cl}(t)$ & $173.14(3)$ & $176.28(6)$ & $169.98(3)$ & $173.35(4)$ & $174.24(5)$ & $174.36(6)$ & $173.39(3)$ \\
\hline $\mathrm{Cl}-\mathrm{M}-\mathrm{Cl}$ & $91.65(4)$ & $92.54(6)$ & $90.82(3)$ & $90.29(4)$ & $90.57(5)$ & $88.37(6)$ & $88.57(3)$ \\
\hline
\end{tabular}

In each structure, the M-S bond is longer then the M-P bond, and the $\mathrm{M}-\mathrm{Cl}$ (trans to $\mathrm{P}$ ) is longer then $\mathrm{M}-\mathrm{Cl}$ (trans to $\mathrm{S}$ ), in agreement with the stronger trans-effect of phosphine donors compared with thioethers. The Pt-P bond is longer in the $t$ Bu derivative $(\mathbf{3 c})$, whereas the $\mathrm{Pd}-\mathrm{P}$ bond is longer in the $\mathrm{Ph}$ derivative (2a). On the other hand, the Pt-S bond is longest in the Et complex (3b) and the $\mathrm{Pd}-\mathrm{S}$ bond is longest in the $t \mathrm{Bu}$ derivative $(\mathbf{2 c})$. An interpretation of these trends does not appear straightforward. The Pt-P distance in compound 3c is in fact significantly longer than all those previously reported for this family of compounds (2.208 $\AA$ for $\mathbf{J}$ [38], 2.2209(1) $\AA$ for $\mathbf{K}$ [39], $2.210 \AA$ for $\mathbf{L}$ [40]), whereas the PdP distances for compounds $\mathbf{2}$ are within the previously established range for compounds A-I (2.228-2.237 ̊). The Pt-S distances are also within the previously established range (2.256$2.293 \AA$ ). On the other hand, all Pd-S distances in compounds 2 are slightly longer than those previously reported for compounds A-I (in the 2.257- 2.296 ̊ range).

The $\mathrm{Pd}-\mathrm{Cl}$ and $\mathrm{Pt}-\mathrm{Cl}$ distances are very close to those of the similar compounds already described in the literature. When comparing the related $\mathrm{Pd}$ and $\mathrm{Pt}$ complexes, the $\mathrm{M}-\mathrm{Cl}$ distances are essentially identical, whereas the M-P and M-S bonds are generally shorter for the heavier Pt metal (exception made for the M-P bond in $\mathbf{2 / 3 c}$ ). A similar observation was made by Carretero for the platinum complex $\mathbf{K}$ and for its palladium analogue [39].

The P-M-S angle varies in the same order for both systems $(\mathbf{b}<\mathbf{a}<\mathbf{c})$, possibly resulting from a growing steric repulsion between the sulphur substituent and the pseudo-axial phosphine $\mathrm{Ph}$ group. In the previously reported compounds, this angle spans a range covering the observed values for compounds 2 (90.0-97. $8^{\circ}$ for A-I), whereas it is smaller than the observed values for compounds $3\left(87-91^{\circ}\right.$ for $\left.\mathbf{J}-\mathbf{L}\right)$. The angle is smaller for the $\mathrm{Pd}$ 
complexes with respect to the Pt analogues, as a consequence of the longer Pd-P and Pd-S distances.

It is worth pointing out that the bulky tert-butyl substituent on the $S$ atom apparently affects the conformation of the Pt complex. Indeed, upon considering the folding between the two planes defined by the substituted $\mathrm{Cp}$-ring and the square $\mathrm{MPSCl}_{2}$ plane, the $\mathrm{CpFe}$ moiety is placed inside the fold for compounds $\mathbf{2 a}, \mathbf{2 b}, \mathbf{2 c}, \mathbf{3 a}, \mathbf{3 b}$ and $\mathbf{3 d}$ and outside for $\mathbf{3 c}$ (see Figures). This change strongly influences the value of the dihedral angle between the two planes, which is much lower in $\mathbf{3 c}, 27.7(1)^{\circ}$, than in the 6 other complex where it ranges from 38.13(6) to $48.21(7)^{\circ}$. The conformation observed in compound $\mathbf{2} \mathbf{c}$ is different than in $\mathbf{3 c}$ and identical to that observed in the other complexes. In this case, the bulky tert-butyl group appear to induce a strong deformation of the square plane, with the two $\mathrm{Cl}$ atoms being pushed away from the mean plane [by -0.666(3) $\AA$ for $\mathrm{Cl} 1$ and $0.332(3) \AA$ for $\mathrm{Cl} 2]$. The molecular geometry may be considered as lying on the isomerization coordinate leading from square planar to tetrahedral [41].

\section{Conclusion}

For the four $(\mathrm{P}, \mathrm{S})$ ligands studied, only monomeric $\mathrm{Pd}(\mathrm{II})$ or $\mathrm{Pt}(\mathrm{II})$ complexes with both $\mathrm{P}$ and $\mathrm{S}$ atoms coordinated on the same metal in a cis geometry were obtained upon reaction with $\mathrm{PdCl}_{2}\left(\mathrm{CH}_{3} \mathrm{CN}\right)_{2}$ or $\mathrm{PtCl}_{2}\left(\mathrm{CH}_{3} \mathrm{CN}\right)_{2}$. For each complex, a slightly distorted square-planar geometry was observed. In addition, only one single diasteroisomer was obtained (both in solution and in solid state) indicating a perfect control of the absolute configuration of the coordinated sulfur atom by the planar chirality of the ferrocene moiety. These observations are of particular interest for the ongoing studies of this type of complexes in asymmetric catalysis. 


\section{Experimental section}

\section{General.}

All reactions were carried out under dry argon by using Schlenk glassware and vacuum line techniques. Solvents were freshly distilled from standard drying agents. ${ }^{1} \mathrm{H},{ }^{13} \mathrm{C}\left\{{ }^{1} \mathrm{H},{ }^{31} \mathrm{P}\right\}$, ${ }^{31} \mathrm{P}\left\{{ }^{1} \mathrm{H}\right\}$ and ${ }^{195} \mathrm{Pt}\left\{{ }^{1} \mathrm{H}\right\}$ NMR spectra were recorded on a Bruker WMX 400 instrument operating at 400,162,100, and $86 \mathrm{MHz}$ respectively. Chemical shifts are reported in parts per million (ppm) relative to $\mathrm{Me}_{4} \mathrm{Si}\left({ }^{1} \mathrm{H}\right.$ and $\left.{ }^{13} \mathrm{C}\right), 85 \% \mathrm{H}_{3} \mathrm{PO}_{4}\left({ }^{31} \mathrm{P}\right)$ or aqueous solution of $\mathrm{Na}_{2} \mathrm{PtCl}_{6}\left({ }^{195} \mathrm{Pt}\right)$. Mass spectra were obtained on a Nermag R10-10 instrument (DCI, FAB) and on a Applied Biosystem API 365 instrument (APCI). Dichlorobis(acetonitrile)palladium(II) and dichlorobis(acetonitrile)platinum(II) were purchased form Strem and used without further purification.

Palladium-dichloro(2-diphenylphosphino-(phenylthiomethyl)ferrocene), 2a. The thioether $1 \mathrm{a}(74 \mathrm{mg}, 0.15 \mathrm{mmol})$ and compound $\mathrm{PdCl}_{2}\left(\mathrm{CH}_{3} \mathrm{CN}\right)_{2}(39 \mathrm{mg}, 0.15 \mathrm{mmol})$ were disolved in $10 \mathrm{~mL}$ of dry dichloromethane under argon. After stirring for two hours at room temperature, the solvent was evaporated and the resulting red solid was washed with dry pentane. Yield: $88 \mathrm{mg}(87 \%) .{ }^{1} \mathrm{H}$ NMR $\left(500 \mathrm{mHz}, \mathrm{CDCl}_{3}\right) \delta(\mathrm{ppm}): 7.84\left(1 \mathrm{H}, \mathrm{d}, \mathrm{J}_{\mathrm{HP}}=12\right.$ $\left.\mathrm{Hz}, \mathrm{PPh}_{2}\right) ; 7.83\left(1 \mathrm{H}, \mathrm{d}, \mathrm{J}_{\mathrm{HP}}=12 \mathrm{~Hz}, \mathrm{PPh}_{2}\right) ; 7.72\left(1 \mathrm{H}, \mathrm{d}, \mathrm{J}_{\mathrm{HP}}=12 \mathrm{~Hz}, \mathrm{PPh}_{2}\right) ; 7.71\left(1 \mathrm{H}, \mathrm{d}, \mathrm{J}_{\mathrm{HP}}\right.$ $\left.=12 \mathrm{~Hz}, \mathrm{PPh}_{2}\right) ; 7.61-7.49(6 \mathrm{H}, \mathrm{m}, \mathrm{Ph}) ; 7.37-7.29(5 \mathrm{H}, \mathrm{m}, \mathrm{Ph}) ; 4.70(1 \mathrm{H}, \mathrm{s}$ large, subst $\mathrm{Cp}) ;$ $4.68(5 \mathrm{H}, \mathrm{s}, \mathrm{Cp}) ; 4.45(1 \mathrm{H}, \mathrm{s}$ large, subst $\mathrm{Cp}) ; 4.08\left(1 \mathrm{H}, \mathrm{d}(\mathrm{AB}), \mathrm{J}_{\mathrm{HH}}=10 \mathrm{~Hz}, \mathrm{C}_{2}-\mathrm{Cp}\right) ; 3.67$ $\left(1 \mathrm{H}\right.$, s large, subst Cp); $3.45\left(1 \mathrm{H}, \mathrm{d}(\mathrm{AB}), \mathrm{J}_{\mathrm{HH}}=10 \mathrm{~Hz}, \mathrm{C}_{2}-\mathrm{Cp}\right) .{ }^{13} \mathrm{C}\left\{{ }^{1} \mathrm{H}\right\} \mathrm{NMR}(500 \mathrm{mHz}$, $\left.\mathrm{CDCl}_{3}\right) \delta(\mathrm{ppm}): 134.2\left(\mathrm{~d}, \mathrm{~J}_{\mathrm{CP}}=11 \mathrm{~Hz}, \mathrm{mPPh}\right) ; 133.5\left(\mathrm{~d}, \mathrm{~J}_{\mathrm{CP}}=11 \mathrm{~Hz}, \mathrm{mPPh}\right) ; 133.3(\mathrm{~s}$, quat $\mathrm{SPh}) ; 132.9(\mathrm{~s}, \mathrm{o} / \mathrm{m} \mathrm{SPh}) ; 132.2\left(\mathrm{~d}, \mathrm{~J}_{\mathrm{CP}}=3 \mathrm{~Hz}, \mathrm{p} \mathrm{PPh}_{2}\right) ; 131.7\left(\mathrm{~d}, \mathrm{~J}_{\mathrm{CP}}=3 \mathrm{~Hz}, \mathrm{p} \mathrm{PPh}\right.$ ); 130.5 (s, p SPh); 129.5 (s, m/o SPh); 129.4 (d, J $\left.\mathrm{J}_{\mathrm{CP}}=11 \mathrm{~Hz}, \mathrm{o} \mathrm{PPh}_{2}\right) ; 128.5\left(\mathrm{~d}, \mathrm{~J}_{\mathrm{CP}}=51 \mathrm{~Hz}\right.$ quat $\left.\mathrm{PPh}_{2}\right) ; 127.9\left(\mathrm{~d}, \mathrm{~J}_{\mathrm{CP}}=12 \mathrm{~Hz}, \mathrm{o} \mathrm{PPh}_{2}\right) ; 127.4\left(\mathrm{~d}, \mathrm{~J}_{\mathrm{CP}}=70 \mathrm{~Hz}\right.$, quat $\left.\mathrm{PPh}_{2}\right) ; 84.3\left(\mathrm{~d}, \mathrm{~J}_{\mathrm{CP}}=16\right.$ 
Hz, quat Cp); $75.3\left(\mathrm{~d}, \mathrm{~J}_{\mathrm{CP}}=7 \mathrm{~Hz}\right.$, subst $\left.\mathrm{Cp}\right) ; 74.8$ (d, $\mathrm{J}_{\mathrm{CP}}=4 \mathrm{~Hz}$, subst $\left.\mathrm{Cp}\right) ; 71.5(\mathrm{~s}, \mathrm{Cp}) ; 69.5$ $\left(\mathrm{d}, \mathrm{J}_{\mathrm{CP}}=7 \mathrm{~Hz}\right.$, subst Cp); $64.6\left(\mathrm{~d}, \mathrm{~J}_{\mathrm{CP}}=62 \mathrm{~Hz}\right.$, quat $\left.\mathrm{Cp}\right) ; 39.4\left(\mathrm{~d}, \mathrm{~J}_{\mathrm{CP}}=4 \mathrm{~Hz}, \mathrm{CH}_{2}-\mathrm{Cp}\right) .{ }^{31} \mathrm{P}$ NMR (500 mHz, $\left.\mathrm{CDCl}_{3}\right) \delta(\mathrm{ppm}): 20.9 ; \mathrm{MS}\left(\mathrm{DCI} ; \mathrm{NH}_{3}\right): \mathrm{m} / \mathrm{z}=688\left(\mathrm{M}+\mathrm{NH}_{4} .28 \%\right)$.

Palladium-dichloro(2-diphenylphosphino-(ethylthiomethyl)ferrocene), 2 b. By following the same procedure described in the previous section, the red product was obtained starting from the thioether $\mathbf{1 b}(100 \mathrm{mg}, 0.225 \mathrm{mmol})$ and $\mathrm{PdCl}_{2}\left(\mathrm{CH}_{3} \mathrm{CN}\right)_{2}(58 \mathrm{mg}, 0.225$ mmol). Yield: $113 \mathrm{mg}(81 \%) .{ }^{1} \mathrm{H}$ NMR $\left(500 \mathrm{mHz}, \mathrm{CDCl}_{3}\right) \delta(\mathrm{ppm}): 7.72-7.53(6 \mathrm{H}, \mathrm{m}, \mathrm{Ph})$; 751-7.41 (4H, m, Ph); $4.63(5 \mathrm{H}, \mathrm{s}, \mathrm{Cp}) ; 4.56(1 \mathrm{H}, \mathrm{s}$ large, subst Cp); $4.41(1 \mathrm{H}, \mathrm{s}$ large, subst $\mathrm{Cp}) ; 3.78\left(1 \mathrm{H}, \mathrm{d}(\mathrm{AB}), \mathrm{J}_{\mathrm{HH}}=12 \mathrm{~Hz}, \underline{\mathrm{CH}}_{2}-\mathrm{Cp}\right) ; 3.53(1 \mathrm{H}, \mathrm{s}$ large, subst $\mathrm{Cp}) ; 3.29\left(1 \mathrm{H}, \mathrm{d}, \mathrm{J}_{\mathrm{HH}}=\right.$ $\left.12 \mathrm{~Hz}, \underline{\mathrm{C}}_{2}-\mathrm{Cp}\right) ; 3.24\left(2 \mathrm{H}, \mathrm{q}, \mathrm{J}_{\mathrm{HH}}=7 \mathrm{~Hz}, \mathrm{C}_{2}-\mathrm{CH}_{3}\right) ; 1.38\left(3 \mathrm{H}, \mathrm{t}, \mathrm{J}_{\mathrm{HH}}=7 \mathrm{~Hz}, \mathrm{CH}_{2}-\underline{\mathrm{C}}_{3}\right)$. ${ }^{13} \mathrm{C}\left\{{ }^{1} \mathrm{H}\right\}$ NMR $\left(500 \mathrm{mHz}, \mathrm{CDCl}_{3}\right) \delta(\mathrm{ppm}): 134.1\left(\mathrm{~d}, \mathrm{~J}_{\mathrm{CP}}=10 \mathrm{~Hz}, \mathrm{mPPh}_{2}\right) ; 133.2\left(\mathrm{~d}, \mathrm{~J}_{\mathrm{CP}}=11\right.$ $\mathrm{Hz}, \mathrm{mPPh}) ; 131.8\left(\mathrm{~d}, \mathrm{~J}_{\mathrm{CP}}=3 \mathrm{~Hz}, \mathrm{p} \mathrm{PPh}_{2}\right) ; 131.6\left(\mathrm{~d}, \mathrm{~J}_{\mathrm{CP}}=3 \mathrm{~Hz}, \mathrm{p} \mathrm{PPh}\right) ; 129.0\left(\mathrm{~d}, \mathrm{~J}_{\mathrm{CP}}=11\right.$ $\mathrm{Hz}$, o $\left.\mathrm{PPh}_{2}\right) ; 128.5\left(\mathrm{~d}, \mathrm{~J}_{\mathrm{CP}}=49 \mathrm{~Hz}\right.$, quat $\left.\mathrm{PPh}_{2}\right) ; 127.9\left(\mathrm{~d}, \mathrm{~J}_{\mathrm{CP}}=71 \mathrm{~Hz}\right.$, quat $\left.\mathrm{PPh}_{2}\right) ; 127.6(\mathrm{~d}$, $\mathrm{J}_{\mathrm{CP}}=12 \mathrm{~Hz}$, o $\left.\mathrm{PPh}_{2}\right) ; 84.4\left(\mathrm{~d}, \mathrm{~J}_{\mathrm{CP}}=16 \mathrm{~Hz}\right.$, quat $\left.\mathrm{Cp}\right) ; 75.7\left(\mathrm{~d}, \mathrm{~J}_{\mathrm{CP}}=6 \mathrm{~Hz}\right.$, subst Cp); $74.2(\mathrm{~d}$, $\mathrm{J}_{\mathrm{CP}}=4 \mathrm{~Hz}$, subst Cp); $71.4(\mathrm{~s}, \mathrm{Cp}) ; 69.3\left(\mathrm{~d}, \mathrm{~J}_{\mathrm{CP}}=7 \mathrm{~Hz}\right.$, subst $\left.\mathrm{Cp}\right) ; 64.6\left(\mathrm{~d}, \mathrm{~J}_{\mathrm{CP}}=64 \mathrm{~Hz}\right.$, quat $\mathrm{Cp}) ; 35.3\left(\mathrm{~s}, \underline{\mathrm{CH}}_{2}-\mathrm{CH}_{3}\right) ; 31.5\left(\mathrm{~d}, \mathrm{~J}_{\mathrm{CP}}=4 \mathrm{~Hz}, \mathrm{CH}_{2}-\mathrm{Cp}\right) ; 13.0\left(\mathrm{~s}, \mathrm{CH}_{2}-\underline{\mathrm{CH}}_{3}\right) .{ }^{31} \mathrm{P}$ NMR (500 $\left.\mathrm{mHz}, \mathrm{CDCl}_{3}\right) \delta(\mathrm{ppm}): 21.2 . \mathrm{MS}\left(\mathrm{DCI} ; \mathrm{NH}_{3}\right): \mathrm{m} / \mathrm{z}=640\left(\mathrm{M}+\mathrm{NH}_{4} .19 \%\right)$.

Palladium-dichloro(2-diphenylphosphino-(tert-butylthiomethyl)ferrocene), 2c. By following the same procedure used for the preparation of $\mathbf{2 a}$, the red product $\mathbf{2} \mathbf{c}$ was obtained starting from the thioether $\mathbf{1 a}(204 \mathrm{mg}, 0.43 \mathrm{mmol})$ and $\mathrm{PdCl}_{2}\left(\mathrm{CH}_{3} \mathrm{CN}\right)_{2}(112 \mathrm{mg}, 0.43$ mmol). Yield: $212 \mathrm{mg}(77 \%) .{ }^{1} \mathrm{H} \mathrm{NMR}\left(500 \mathrm{mHz}, \mathrm{CDCl}_{3}\right) \delta(\mathrm{ppm}): 7.66\left(1 \mathrm{H}, \mathrm{d}, \mathrm{J}_{\mathrm{HP}}=12 \mathrm{~Hz}\right.$, $\left.\mathrm{PPh}_{2}\right) ; 7.65\left(1 \mathrm{H}, \mathrm{d}, \mathrm{J}_{\mathrm{HP}}=13 \mathrm{~Hz}, \mathrm{PPh}_{2}\right) ; 7.62\left(1 \mathrm{H}, \mathrm{d}, \mathrm{J}_{\mathrm{HP}}=13 \mathrm{~Hz}, \mathrm{PPh}_{2}\right) ; 7.60\left(1 \mathrm{H}, \mathrm{d}, \mathrm{J}_{\mathrm{HP}}=\right.$ $\left.13 \mathrm{~Hz}, \mathrm{PPh}_{2}\right) ; 7.57-7.46\left(4 \mathrm{H}, \mathrm{m}, \mathrm{PPh}_{2}\right) ; 7.42\left(2 \mathrm{H}, \mathrm{dt}, \mathrm{J}_{\mathrm{HH}}=8 \mathrm{~Hz}, \mathrm{~J}_{\mathrm{HP}}=3 \mathrm{~Hz}, \mathrm{PPh}_{2}\right) ; 4.74(5 \mathrm{H}$, s, Cp); $4.71\left(1 \mathrm{H}, \mathrm{s}\right.$ large, subst Cp); $4.42(1 \mathrm{H}, \mathrm{s}$ large, subst $\mathrm{Cp}) ; 3.78\left(1 \mathrm{H}, \mathrm{d}(\mathrm{AB}), \mathrm{J}_{\mathrm{HH}}=11\right.$ $\left.\mathrm{Hz}, \underline{\mathrm{C}}_{2}-\mathrm{Cp}\right) ; 3.52\left(1 \mathrm{H}, \mathrm{s}\right.$ large, subst Cp); $3.20\left(1 \mathrm{H}, \mathrm{d}(\mathrm{AB}), \mathrm{J}_{\mathrm{HH}}=12 \mathrm{~Hz}, \underline{\mathrm{C}}_{2}-\mathrm{Cp}\right), 1.46(9 \mathrm{H}$, 
s, $\left.\mathrm{C}\left(\mathrm{CH}_{3}\right)_{3}\right) .{ }^{13} \mathrm{C}\left\{{ }^{1} \mathrm{H}\right\} \mathrm{NMR}\left(500 \mathrm{mHz}, \mathrm{CDCl}_{3}\right) \delta(\mathrm{ppm}): 134.0\left(\mathrm{~d}, \mathrm{~J}_{\mathrm{CP}}=10 \mathrm{~Hz}, \mathrm{PPh}_{2}\right) ; 133.0$ $\left(\mathrm{d}, \mathrm{J}_{\mathrm{CP}}=11 \mathrm{~Hz}, \mathrm{PPh}_{2}\right) ; 132.1\left(\mathrm{~d}, \mathrm{~J}_{\mathrm{CP}}=3 \mathrm{~Hz}, \mathrm{p} \mathrm{PPh}_{2}\right) ; 131.4\left(\mathrm{~d}, \mathrm{~J}_{\mathrm{CP}}=3 \mathrm{~Hz}, \mathrm{p} \mathrm{PPh}_{2}\right) ; 129.3(\mathrm{~d}$, $\left.\mathrm{J}_{\mathrm{CP}}=11 \mathrm{~Hz}, \mathrm{PPh}_{2}\right) ; 128.3\left(\mathrm{~d}, \mathrm{~J}_{\mathrm{CP}}=49 \mathrm{~Hz}\right.$, quat $\left.\mathrm{PPh}_{2}\right) ; 128.2\left(\mathrm{~d}, \mathrm{~J}_{\mathrm{CP}}=71 \mathrm{~Hz}\right.$, quat $\left.\mathrm{PPh}_{2}\right)$; $127.7\left(\mathrm{~d}, \mathrm{~J}_{\mathrm{CP}}=12 \mathrm{~Hz}, \mathrm{PPh}_{2}\right) ; 84.9\left(\mathrm{~d}, \mathrm{~J}_{\mathrm{CP}}=17 \mathrm{~Hz}\right.$, quat $\left.\mathrm{Cp}\right) ; 75.3\left(\mathrm{~d}, \mathrm{~J}_{\mathrm{CP}}=7 \mathrm{~Hz}\right.$, subst $\left.\mathrm{Cp}\right)$; $75.1\left(\mathrm{~d}, \mathrm{~J}_{\mathrm{CP}}=4 \mathrm{~Hz}\right.$, subst Cp); $71.6(\mathrm{~s}, \mathrm{Cp}) ; 69.6\left(\mathrm{~d}, \mathrm{~J}_{\mathrm{CP}}=7 \mathrm{~Hz}\right.$, subst Cp); $63.7\left(\mathrm{~d}, \mathrm{~J}_{\mathrm{CP}}=63\right.$ $\mathrm{Hz}$, quat $\mathrm{Cp}$ ); 56.1 (s, $\left.\underline{\mathrm{C}}\left(\mathrm{CH}_{3}\right)_{3}\right) ; 30.9$ (s, $\left.\mathrm{C}\left(\underline{\mathrm{CH}}_{3}\right)_{3}\right) ; 28.0$ (d, J $\mathrm{CP}=4 \mathrm{~Hz}, \underline{\left.\mathrm{CH}_{2}-\mathrm{Cp}\right) .}{ }^{31} \mathrm{P} \mathrm{NMR}$ $\left(500 \mathrm{mHz}, \mathrm{CDCl}_{3}\right) \delta(\mathrm{ppm}): 22.4 . \mathrm{MS}\left(\mathrm{DCI} ; \mathrm{NH}_{3}\right): \mathrm{m} / \mathrm{z}=668\left(\mathrm{M}+\mathrm{NH}_{4} .16 \%\right)$.

Platinum-dichloro(2-diphenylphosphino-(phenylthiomethyl)ferrocene), 3a. The thioether 1a $(127 \mathrm{mg}, 0.258 \mathrm{mmol})$ and $\mathrm{PtCl}_{2}\left(\mathrm{CH}_{3} \mathrm{CN}\right)_{2}(75 \mathrm{mg}, 0.216 \mathrm{mmol})$ were disolved in $20 \mathrm{~mL}$ of dry toluene under argon. After one hour of reflux, the orange solid which formed was filtered and washed with dry pentane, yielding the product as an orange solid (154 mg, 95\% yield). ${ }^{1} \mathrm{H}$ NMR $\left(500 \mathrm{mHz}, \mathrm{CDCl}_{3}\right) \delta(\mathrm{ppm}): 7.81-7.65(6 \mathrm{H}, \mathrm{m}, \mathrm{Ph}) ; 7.55-7.40(6 \mathrm{H}, \mathrm{m}$, $\mathrm{Ph})$; 7.41-7.30 (3H, m, Ph); $4.60(5 \mathrm{H}, \mathrm{s}, \mathrm{Cp}) ; 4.58(1 \mathrm{H}, \mathrm{m}$, subst $\mathrm{Cp}) ; 4.38\left(1 \mathrm{H}, \mathrm{td}, \mathrm{J}_{\mathrm{HH}}=\right.$ $2.5 \mathrm{~Hz}, \mathrm{~J}_{\mathrm{HP}}=1 \mathrm{~Hz}$, subst $\left.\mathrm{Cp}\right) ; 4.15\left(1 \mathrm{H}, \mathrm{dd}(\mathrm{ABX}), \mathrm{J}_{\mathrm{HH}}=13 \mathrm{~Hz}, \mathrm{~J}_{\mathrm{HP}}=2 \mathrm{~Hz}, \underline{C}_{2}-\mathrm{Cp}\right) ; 3.67$ $\left(1 \mathrm{H}, \mathrm{m}\right.$, subst Cp); $3.61\left(1 \mathrm{H}, \mathrm{d}(\mathrm{AB}), \mathrm{J}_{\mathrm{HH}}=13 \mathrm{~Hz}, \mathrm{C}_{2}-\mathrm{Cp}\right) .{ }^{13} \mathrm{C}\left\{{ }^{1} \mathrm{H}\right\} \mathrm{NMR}\left(500 \mathrm{mHz}, \mathrm{CDCl}_{3}\right)$ $\delta(\mathrm{ppm}): 134.6\left(\mathrm{~d}, \mathrm{~J}_{\mathrm{CP}}=11 \mathrm{~Hz}, \mathrm{~m} \mathrm{PPh}_{2}\right) ; 133.7\left(\mathrm{~d}, \mathrm{~J}_{\mathrm{CP}}=11 \mathrm{~Hz}, \mathrm{~m} \mathrm{PPh}_{2}\right) ; 133.3(\mathrm{~s}, \mathrm{o} \mathrm{SPh})$; 133.2 (s, quat $\mathrm{SPh}) ; 132.1\left(\mathrm{~d}, \mathrm{~J}_{\mathrm{CP}}=2 \mathrm{~Hz}, \mathrm{p} \mathrm{PPh}_{2}\right) ; 132.0\left(\mathrm{~d}, \mathrm{~J}_{\mathrm{CP}}=2 \mathrm{~Hz}, \mathrm{p} \mathrm{PPh}_{2}\right) ; 131.2(\mathrm{~s}, \mathrm{p}$ $\mathrm{SPh}) ; 129.8$ (s, m SPh); $129.5\left(\mathrm{~d}, \mathrm{~J}_{\mathrm{CP}}=11 \mathrm{~Hz}, \mathrm{o} \mathrm{PPh}_{2}\right) ; 129.0\left(\mathrm{~d}, \mathrm{~J}_{\mathrm{CP}}=60 \mathrm{~Hz}\right.$, quat $\left.\mathrm{PPh}_{2}\right)$; $128.2\left(\mathrm{~d}, \mathrm{~J}_{\mathrm{CP}}=12 \mathrm{~Hz}, o \mathrm{PPh}_{2}\right) ; 127.1\left(\mathrm{~d}, \mathrm{~J}_{\mathrm{CP}}=75 \mathrm{~Hz}\right.$, quat $\left.\mathrm{PPh}_{2}\right) ; 84.3\left(\mathrm{~d}, \mathrm{~J}_{\mathrm{CP}}=14 \mathrm{~Hz}\right.$, quat $\mathrm{Cp}) ; 75.8\left(\mathrm{~d}, \mathrm{~J}_{\mathrm{CP}}=7 \mathrm{~Hz}\right.$, subst Cp); $74.6\left(\mathrm{~d}, \mathrm{~J}_{\mathrm{CP}}=12 \mathrm{~Hz}\right.$, subst Cp); $71.9(\mathrm{~s}, \mathrm{Cp}) ; 69.3\left(\mathrm{~d}, \mathrm{~J}_{\mathrm{CP}}\right.$ $=7 \mathrm{~Hz}$, subst $\mathrm{Cp}) ; 65.9\left(\mathrm{~d}, \mathrm{~J}_{\mathrm{CP}}=73 \mathrm{~Hz}\right.$, quat $\left.\mathrm{Cp}\right) ; 41.5\left(\mathrm{~d}, \mathrm{~J}_{\mathrm{CP}}=2 \mathrm{~Hz}, \mathrm{CH}_{2}-\mathrm{Cp}\right) .{ }^{31} \mathrm{P}$ NMR $\left(500 \mathrm{mHz}, \mathrm{CDCl}_{3}\right) \delta(\mathrm{ppm}):-0.8$ (satellites $\left.{ }^{195} \mathrm{Pt}: \mathrm{J}_{\mathrm{PPt}}=3608 \mathrm{~Hz}\right) .{ }^{195} \mathrm{Pt} \mathrm{NMR}(500 \mathrm{mHz}$, $\left.\mathrm{CDCl}_{3}\right) \delta(\mathrm{ppm}):-4093\left(\mathrm{~J}_{\mathrm{PtP}}=3608 \mathrm{~Hz}\right) . \mathrm{MS}\left(\mathrm{DCI} ; \mathrm{NH}_{3}\right): \mathrm{m} / \mathrm{z}=776\left(\mathrm{M}+\mathrm{NH}_{4} .100 \%\right)$.

Platinum-dichloro(2-diphenylphosphino-(ethylthiomethyl)ferrocene), $3 b . \quad$ By following the same procedure described in the previous section, the orange product was 
obtained after a $3 \mathrm{~h}$ reflux starting from the thioether $\mathbf{1 b}(350 \mathrm{mg}, 0.79 \mathrm{mmol})$ and $\mathrm{PtCl}_{2}\left(\mathrm{CH}_{3} \mathrm{CN}\right)_{2}(240 \mathrm{mg}, 0.70 \mathrm{mmol})$. Yield; $440 \mathrm{mg}(89 \%) .{ }^{1} \mathrm{H} \mathrm{NMR}\left(500 \mathrm{mHz}, \mathrm{CDCl}_{3}\right)$ $\delta(\mathrm{ppm}):$ 7.72-7.63 (4H, m, Ph); 754-7.41 (6H, m, Ph); 4.67 (1H, s large, subst Cp); $4.53(5 \mathrm{H}$, s, Cp); $4.39(1 \mathrm{H}, \mathrm{s}$ large, subst $\mathrm{Cp}) ; 3.96\left(1 \mathrm{H}, \mathrm{d}(\mathrm{AB}), \mathrm{J}_{\mathrm{HH}}=12 \mathrm{~Hz}, \mathrm{C}_{2}-\mathrm{Cp}\right) ; 3.63(1 \mathrm{H}, \mathrm{s}$ large, subst Cp); 3.39-3.29 (3H, m, $\underline{\mathrm{C}}_{2}-\mathrm{Cp}$ et $\left.\underline{\mathrm{C}}_{2}-\mathrm{CH}_{3}\right) ; 1.35\left(3 \mathrm{H}, \mathrm{t}, \mathrm{J}_{\mathrm{HH}}=7 \mathrm{~Hz}, \mathrm{CH}_{2}-\mathrm{C}_{3}\right)$. ${ }^{13} \mathrm{C}\left\{{ }^{1} \mathrm{H}\right\}$ NMR $\left(500 \mathrm{mHz}, \mathrm{CDCl}_{3}\right) \delta(\mathrm{ppm}): 134.2\left(\mathrm{~d}, \mathrm{~J}_{\mathrm{CP}}=11 \mathrm{~Hz}, \mathrm{mPPh} 2\right) ; 133.3\left(\mathrm{~J}_{\mathrm{CP}}=11\right.$ $\mathrm{Hz}, \mathrm{mPPh}) ; 131.6\left(\mathrm{~d}, \mathrm{~J}_{\mathrm{CP}}=2 \mathrm{~Hz}, \mathrm{p} \mathrm{PPh}_{2}\right) ; 131.5\left(\mathrm{~d}, \mathrm{~J}_{\mathrm{CP}}=2 \mathrm{~Hz}, \mathrm{pPPh} 2\right) ; 128.9\left(\mathrm{~d}, \mathrm{~J}_{\mathrm{CP}}=11\right.$ $\left.\mathrm{Hz}, \mathrm{o} \mathrm{PPh}_{2}\right) ; 128.5\left(\mathrm{~d}, \mathrm{~J}_{\mathrm{CP}}=62 \mathrm{~Hz}\right.$, quat $\left.\mathrm{PPh}_{2}\right) ; 127.7\left(\mathrm{~d}, \mathrm{~J}_{\mathrm{CP}}=11 \mathrm{~Hz}, \mathrm{o} \mathrm{PPh}_{2}\right) ; 126.8\left(\mathrm{~d}, \mathrm{~J}_{\mathrm{CP}}=\right.$ $76 \mathrm{~Hz}$, quat $\left.\mathrm{PPh}_{2}\right) ; 84.3\left(\mathrm{~d}, \mathrm{~J}_{\mathrm{CP}}=15 \mathrm{~Hz}\right.$, quat $\left.\mathrm{Cp}\right) ; 75.3\left(\mathrm{~d}, \mathrm{~J}_{\mathrm{CP}}=7 \mathrm{~Hz}\right.$, subst $\left.\mathrm{Cp}\right) ; 73.9\left(\mathrm{~d}, \mathrm{~J}_{\mathrm{CP}}=\right.$ 5Hz, subst Cp); $71.4(\mathrm{~s}, \mathrm{Cp}) ; 68.8\left(\mathrm{~d}, \mathrm{~J}_{\mathrm{CP}}=8 \mathrm{~Hz}\right.$, subst $\left.\mathrm{Cp}\right) ; 65.8\left(\mathrm{~d}, \mathrm{~J}_{\mathrm{CP}}=73 \mathrm{~Hz}\right.$, quat $\left.\mathrm{Cp}\right)$; $35.5\left(\mathrm{~s}, \underline{\mathrm{CH}}_{2}-\mathrm{CH}_{3}\right) ; 31.5\left(\mathrm{~d}, \mathrm{~J}_{\mathrm{CP}}=3 \mathrm{~Hz}, \mathrm{CH}_{2}-\mathrm{Cp}\right) ; 13.7\left(\mathrm{~s}, \mathrm{CH}_{2}-\underline{C H}_{3}\right) .{ }^{31} \mathrm{P}$ NMR $(500 \mathrm{mHz}$, $\left.\mathrm{CDCl}_{3}\right) \delta(\mathrm{ppm}):-3.68\left(\right.$ satellites $\left.{ }^{195} \mathrm{Pt}: \mathrm{J}_{\mathrm{PPt}}=3666 \mathrm{~Hz}\right) .{ }^{195} \mathrm{Pt} \mathrm{NMR}\left(500 \mathrm{mHz}, \mathrm{CDCl}_{3}\right)$ $\delta(\mathrm{ppm}):-4110\left(\mathrm{~J}_{\mathrm{PtP}}=3666 \mathrm{~Hz}\right) . \mathrm{MS}\left(\mathrm{DCI} ; \mathrm{NH}_{3}\right): \mathrm{m} / \mathrm{z}=728\left(\mathrm{M}+\mathrm{NH}_{4} .100 \%\right)$.

Platinum-dichloro(2-diphenylphosphino-(tert-butylthiomethyl)ferrocene), 3c. Вy following the same procedure used for the preparation of $\mathbf{3 a}$, the orange product $\mathbf{3 c}$ was obtained after a $3 \mathrm{~h}$ reflux starting from the thioether 1c $(370 \mathrm{mg}, 0.78 \mathrm{mmol})$ and $\mathrm{PtCl}_{2}\left(\mathrm{CH}_{3} \mathrm{CN}\right)_{2}(240 \mathrm{mg}, 0.70 \mathrm{mmol})$. Yield: $480 \mathrm{mg}(93 \%) .{ }^{1} \mathrm{H} \mathrm{NMR}\left(500 \mathrm{mHz}, \mathrm{CDCl}_{3}\right)$ $\delta(\mathrm{ppm}): 7.69\left(1 \mathrm{H}, \mathrm{d}, \mathrm{J}_{\mathrm{HP}}=13 \mathrm{~Hz}, \mathrm{mPPh}\right) ; 7.67\left(1 \mathrm{H}, \mathrm{d}, \mathrm{J}_{\mathrm{HP}}=13 \mathrm{~Hz}, \mathrm{~m} \mathrm{PPh}_{2}\right) ; 7.59(1 \mathrm{H}, \mathrm{d}$, $\mathrm{J}_{\mathrm{HP}}=13 \mathrm{~Hz}, \mathrm{mPPh}$ ); $7.57\left(1 \mathrm{H}, \mathrm{d}, \mathrm{J}_{\mathrm{HP}}=13 \mathrm{~Hz}, \mathrm{mPPh}\right.$ ); 7.53-7.50 (2H, m, $\left.\mathrm{PPh}_{2}\right) ; 7.46-7.43$ $\left(4 \mathrm{H}, \mathrm{m}, \mathrm{PPh}_{2}\right) ; 4.63(1 \mathrm{H}, \mathrm{s}$ large, subst $\mathrm{Cp}) ; 4.56(5 \mathrm{H}, \mathrm{s}, \mathrm{Cp}) ; 4.38(1 \mathrm{H}, \mathrm{s}$ large, subst $\mathrm{Cp})$; $3.99\left(1 \mathrm{H}, \mathrm{d}(\mathrm{AB}), \mathrm{J}_{\mathrm{HH}}=13 \mathrm{~Hz}, \underline{\mathrm{C}}_{2}-\mathrm{Cp}\right) ; 3.66(1 \mathrm{H}, \mathrm{s}$ large, subst $\mathrm{Cp}) ; 3.39\left(1 \mathrm{H}, \mathrm{d}(\mathrm{AB}), \mathrm{J}_{\mathrm{HH}}=\right.$ $\left.13 \mathrm{~Hz}, \underline{\mathrm{CH}}_{2} \mathrm{Cp}\right) ; 1.42\left(9 \mathrm{H}, \mathrm{s}, \mathrm{C}\left(\mathrm{C}_{3}\right)_{3}\right) .{ }^{13} \mathrm{C}\left\{{ }^{1} \mathrm{H}\right\} \mathrm{NMR}\left(500 \mathrm{mHz}, \mathrm{CDCl}_{3}\right) \delta(\mathrm{ppm}): 134.1(\mathrm{~d}$, $\left.\mathrm{J}_{\mathrm{CP}}=11 \mathrm{~Hz}, \mathrm{mPPh}\right) ; 133.2\left(\mathrm{~d}, \mathrm{~J}_{\mathrm{CP}}=11 \mathrm{~Hz}, \mathrm{mPPh}_{2}\right) ; 131.6\left(\mathrm{~d}, \mathrm{~J}_{\mathrm{CP}}=3 \mathrm{~Hz}, \mathrm{p} \mathrm{PPh}_{2}\right) ; 131.3(\mathrm{~d}$, $\left.\mathrm{J}_{\mathrm{CP}}=3 \mathrm{~Hz}, \mathrm{p} \mathrm{PPh} 2\right) ; 128.9\left(\mathrm{~d}, \mathrm{~J}_{\mathrm{CP}}=11 \mathrm{~Hz}, \mathrm{o} \mathrm{PPh}_{2}\right) ; 128.5\left(\mathrm{~d}, \mathrm{~J}_{\mathrm{CP}}=60 \mathrm{~Hz}\right.$, quat $\left.\mathrm{PPh}_{2}\right) ; 127.7$ $\left(\mathrm{d}, \mathrm{J}_{\mathrm{CP}}=76 \mathrm{~Hz}\right.$, quat $\left.\mathrm{PPh}_{2}\right) ; 127.6\left(\mathrm{~d}, \mathrm{~J}_{\mathrm{CP}}=12 \mathrm{~Hz}\right.$, o $\left.\mathrm{PPh}_{2}\right) ; 84.5\left(\mathrm{~d}, \mathrm{~J}_{\mathrm{CP}}=15 \mathrm{~Hz}\right.$, quat $\left.\mathrm{Cp}\right)$; 
$74.7\left(\mathrm{~d}, \mathrm{~J}_{\mathrm{CP}}=8 \mathrm{~Hz}\right.$, subst Cp); $74.6\left(\mathrm{~d}, \mathrm{~J}_{\mathrm{CP}}=7 \mathrm{~Hz}\right.$, subst Cp); $71.5(\mathrm{~s}, \mathrm{Cp}) ; 68.6\left(\mathrm{~d}, \mathrm{~J}_{\mathrm{CP}}=8 \mathrm{~Hz}\right.$,

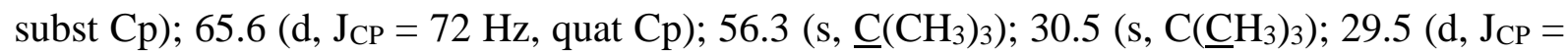
$\left.3 \mathrm{~Hz}, \underline{\mathrm{CH}}_{2}-\mathrm{Cp}\right) ;{ }^{31} \mathrm{P}$ NMR $\left(500 \mathrm{mHz}, \mathrm{CDCl}_{3}\right) \delta(\mathrm{ppm}):-3.18$ (satellites ${ }^{195} \mathrm{Pt}: \mathrm{J}_{\mathrm{PPt}}=3636$ Hz). ${ }^{195} \mathrm{Pt}$ NMR $\left(500 \mathrm{mHz}, \mathrm{CDCl}_{3}\right) \delta(\mathrm{ppm}):-4030\left(\mathrm{~J}_{\mathrm{PtP}}=3636 \mathrm{~Hz}\right) ; \mathrm{MS}\left(\mathrm{DCI} ; \mathrm{NH}_{3}\right): \mathrm{m} / \mathrm{z}=$ $756\left(\mathrm{M}+\mathrm{NH}_{4} .26 \%\right)$

Platinum-dichloro(2-diphenylphosphino-cyclohexylthiomethyl)ferrocene), 3d. By following the same procedure used for the preparation of $\mathbf{3 a}$, the orange product $\mathbf{3 d}$ was obtained after a $1 \mathrm{~h}$ reflux starting from the thioether $1 \mathbf{d}(95 \mathrm{mg}, 0.191 \mathrm{mmol})$ and $\mathrm{PtCl}_{2}\left(\mathrm{CH}_{3} \mathrm{CN}\right)_{2}(56 \mathrm{mg}, 0.173 \mathrm{mmol})$. Yield: $123 \mathrm{mg}(93 \%) .{ }^{1} \mathrm{H} \mathrm{NMR}\left(500 \mathrm{mHz}, \mathrm{CDCl}_{3}\right)$ $\delta(\mathrm{ppm})$ : 7.7-7.45 (10H, m : $\left.\mathrm{PPh}_{2}\right) ; 4.66(1 \mathrm{H}, \mathrm{m}:$ subst Cp); $4.57(5 \mathrm{H}, \mathrm{s}: \mathrm{Cp}) ; 4.41(1 \mathrm{H}, \mathrm{td}$, $\mathrm{J}_{\mathrm{HH}}=2.5 \mathrm{~Hz}$ and $\mathrm{J}_{\mathrm{PH}}=0.9 \mathrm{~Hz}:$ subst $\left.\mathrm{Cp}\right) ; 3.94(1 \mathrm{H}, \mathrm{m}: \mathrm{CH}) ; 3.80\left(1 \mathrm{H}\right.$, dd $(\mathrm{ABX}), \mathrm{J}_{\mathrm{HH}}$ $=12.6 \mathrm{~Hz}$ and $\left.\mathrm{J}_{\mathrm{PH}}=1.8 \mathrm{~Hz}: \mathrm{C}_{2} \mathrm{Cp}\right) ; 3.63(1 \mathrm{H}, \mathrm{m}$ : subst $\mathrm{Cp}) ; 3.30\left(1 \mathrm{H}, \mathrm{d}(\mathrm{AB}), \mathrm{J}_{\mathrm{HH}}=12.6 \mathrm{~Hz}\right.$ : $\left.\underline{\mathrm{CH}}_{2} \mathrm{Cp}\right) ; 2.50(1 \mathrm{H}, \mathrm{m}: \mathrm{Cy}) ; 2.15(1 \mathrm{H}, \mathrm{m}: \mathrm{Cy}) ; 1.85-1.05(8 \mathrm{H}, \mathrm{m}: \mathrm{Cy}) .{ }^{13} \mathrm{C}\left\{{ }^{1} \mathrm{H}\right\} \mathrm{NMR}(500$ $\left.\mathrm{mHz}, \mathrm{CDCl}_{3}\right) \delta(\mathrm{ppm}): 134.3\left(\mathrm{~d}, \mathrm{~J}_{\mathrm{CP}}=10.8 \mathrm{~Hz}: \mathrm{PPh}_{2}\right) ; 133.7\left(\mathrm{~d}, \mathrm{~J}_{\mathrm{CP}}=11.0 \mathrm{~Hz}: \mathrm{PPh}_{2}\right) ; 131.9$ $\left(\mathrm{d}, \mathrm{J}_{\mathrm{CP}}=3.9 \mathrm{~Hz}: \mathrm{PPh}_{2}\right) ; 131.7\left(\mathrm{~d}, \mathrm{~J}_{\mathrm{CP}}=4.0 \mathrm{~Hz}: \mathrm{PPh}_{2}\right) ; 129.2\left(\mathrm{~d}, \mathrm{~J}_{\mathrm{CP}}=11.2 \mathrm{~Hz}: \mathrm{PPh}_{2}\right) ; 128.6$ $\left(\mathrm{d}, \mathrm{J}_{\mathrm{CP}}=61.9 \mathrm{~Hz}:\right.$ quat $\left.\mathrm{PPh}_{2}\right) ; 128.0\left(\mathrm{~d}, \mathrm{~J}_{\mathrm{CP}}=12.1 \mathrm{~Hz}: \mathrm{PPh}_{2}\right) ; 127.4\left(\mathrm{~d}, \mathrm{~J}_{\mathrm{CP}}=75.3 \mathrm{~Hz}:\right.$ quat $\left.\mathrm{PPh}_{2}\right) ; 84.6\left(\mathrm{~d}, \mathrm{~J}_{\mathrm{CP}}=14.7 \mathrm{~Hz}:\right.$ quat $\left.\mathrm{Cp}\right) ; 75.8\left(\mathrm{~d}, \mathrm{~J}_{\mathrm{CP}}=7.1 \mathrm{~Hz}:\right.$ subst $\left.\mathrm{Cp}\right) ; 74.5\left(\mathrm{~d}, \mathrm{~J}_{\mathrm{CP}}=5.4 \mathrm{~Hz}\right.$ : subst Cp); $71.7(\mathrm{~s}: \mathrm{Cp}) ; 69.1\left(\mathrm{~d}, \mathrm{~J}_{\mathrm{CP}}=7.5 \mathrm{~Hz}\right.$ : subst $\left.\mathrm{Cp}\right) ; 65.9\left(\mathrm{~d}, \mathrm{~J}_{\mathrm{CP}}=73.5 \mathrm{~Hz}:\right.$ quat $\left.\mathrm{Cp}\right)$; 52.8 (s : CH); 32.1 (s : Cy); 31.8 (s : Cy); 27.4 (s : Cy); 27.0 (s : Cy); 26.2 (s : $\underline{\mathrm{CH}}_{2} \mathrm{Cp}$ ); 25.5 (s : Cy). ${ }^{31} \mathrm{P}$ NMR $\left(500 \mathrm{mHz}, \mathrm{CDCl}_{3}\right) \delta(\mathrm{ppm}):-0.5$ (satellites $\left.{ }^{195} \mathrm{Pt}: \mathrm{J}_{\mathrm{PPt}}=3660 \mathrm{~Hz}\right) .{ }^{195} \mathrm{Pt}$ NMR $\left(500 \mathrm{mHz}, \mathrm{CDCl}_{3}\right) \delta(\mathrm{ppm}):-4095\left(\mathrm{~d}, \mathrm{~J}_{\mathrm{PPt}}=3660 \mathrm{~Hz}\right)$.

X-ray crystallography. A single crystal of each compound was mounted under inert perfluoropolyether at the tip of a glass fibre and cooled in the cryostream of either an OxfordDiffraction XCALIBUR CCD or a Stoe IPDS diffractometer. Data were collected using the monochromatic MoK $\alpha$ radiation $(\lambda=0.71073)$. 
The structures were solved by direct methods (SIR97) [42] and refined by least-squares procedures on $F^{2}$ using SHELXL-97 [43]. All $\mathrm{H}$ atoms attached to carbon were introduced in idealised positions and treated as riding models in the calculations. In compound $\mathbf{2} \mathbf{b}$, there are two molecules within the asymmetric unit and surprisingly they have opposite planar chirality $(R$ and $S$ ) even though they are not related by any symmetry elements; the Flack's parameter [44] close to 0 is indicative of a non centrosymmetric structure. The drawing of the molecules was realised with the help of ORTEP3 [45]. Crystal data and refinement parameters are shown in .

Crystallographic data (excluding structure factors) have been deposited with the Cambridge Crystallographic Data Centre as supplementary publication no. CCDC 653929 653935. Copies of the data can be obtained free of charge on application to the Director, CCDC, 12 Union Road, Cambridge CB2 1EZ, UK (fax: (+44) 1223-336-033; e-mail: deposit@ccdc.cam.ac.uk).

\section{References}

[1] F. A. Cotton, G. Wilkinson, J. Am. Chem. Soc. 74 (1952) 5764.

[2] F. A. Cotton, J. Organomet. Chem. 637 (2001) 18.

[3] F. Barrière, Encyclopedia of Electrochemistry 7a (2006) 461.

[4] A. Federman Neto, A. C. Pelegrino, V. A. Darin, Trends in Organometallic Chemistry 4 (2002) 147.

[5] T. Chuard, R. Deschenaux, Chimia 57 (2003) 597.

[6] J. J. Wang, L. Wang, W. Q. Wang, T. Chen, Journal of Polymer Materials 22 (2005) 169.

[7] For general reviews about planar chirality, see: (a) K. Schlögl, Topics in Sterochemistry. 1 (1967), 39. (b) K. Schlögl, Topics in Current Chemistry 125 (1984), 27. For reviews about synthesis of enantiomerically pure planar chiral ferrocene derivatives: (c) C. J. Richards, A. J. Locke, Tetrahedron Asymmetry, 9 (1998), 2377. (d) G. G. A. Balavoine, J.-C. Daran, G. Iftime, E. Manoury, C. Moreau-Bossuet, J. Organomet. Chem., 567 (1998) 191. (e) O. Riant, H. B. Kagan, Advances in Asymmetric Synthesis, Hassner, A., Ed; Jai press, 1997, Vol 2, 189. (f) A. Togni, Angew. Chem. Int. Ed. Engl., 35 (1996), 1475.

[8] For reviews about chiral ferrocenyl ligands : (a) R. Gomez Arrayas, J. Adrio, J. C. Carretero, Angew. Chem. Int. Ed. 45 (2006) 7674. (b) R. C. J. Atkinson, V. C. Gibson, N. J. Long, Chem. Soc. Rev. 33 (2004) 313. (c) T. J. Colacot, Chem. Rev. 103 
(2003) 3101. (d) P. Barbaro, C. Bianchini, G. Giambastiani, S. L. Parisel, Coord. Chem. Rev. 248 (2004) 2131.

[9] M. Sawamura, Y. Nakayama, T. Kato, Y. Ito, J. Org. Chem. 60 (1995) 1727.

[10] M. Sawamura, M. Sudoh, Y. Ito, J. Am. Chem. Soc. 118 (1996) 3309.

[11] R. Kuwano, H. Miyazaki, Y. Ito, Chem. Commun. (1998) 71.

[12] R. Kuwano, T. Uemura, M. Saitoh, Y. Ito, Tetrahedron Lett. 40 (1999) 1327.

[13] R. Kuwano, K. Sato, T. Kurokawa, D. Karube, Y. Ito, J. Am. Chem. Soc. 122 (2000) 7614.

[14] H.-U. Blaser, W. Brieden, B. Pugin, F. Spindler, M. Studer, A. Togni, Topics in Catalysis 19 (2002) 3, and references quoted therein.

[15] H.-U. Blaser, Adv. Synth. Catal. 344 (2002) 17, and references quoted therein.

[16] T. Ireland, G. Grossheimann, C. Wieser-Jeunesse, P. Knochel, Angew. Chem., Int. Ed. Engl. 38 (1999) 3212.

[17] M. Lotz, K. Polborn, P. Knochel, Angew. Chem., Int. Ed. Engl. 41 (2002) 4708.

[18] F. Spindler, C. Malan, M. Lotz, M. Kesselgruber, U. Pittelkow, A. Rivas-Nass, O. Briel, H. U. Blaser, Tetrahedron-Asymmetry 15 (2004) 2299.

[19] T. Sturm, W. Weissensteiner, F. Spindler, Adv. Synth. Catal. 345 (2003) 160.

[20] O. B. Sutcliffe, M. R. Bryce, Tetrahedron-Asymmetry 14 (2003) 2297, and references quoted therein.

[21] S. Cabrera, O. G. Mancheno, R. G. Arrayas, I. Alonso, P. Mauleon, J. C. Carretero, Pure Appl. Chem. 78 (2006) 257.

[22] L. Routaboul, S. Vincendeau, J.-C. Daran, E. Manoury, Tetrahedron: Asymmetry 16 (2005) 2685.

[23] For the synthesis of enantiomerically pure synthetic intermediates (2diphenylthiophosphinoferrocenyl)-methanol, see: N. Mateus, L. Routaboul, J.-C. Daran, E. Manoury, J. Organomet. Chem., 691 (2006) 2297.

[24] R. Malacea, E. Manoury, L. Routaboul, J.-C. Daran, R. Poli, J. P. Dunne, A. C. Withwood, C. Godard, S. B. Duckett, Eur. J. Inorg. Chem. (2006) 1803-1816.

[25] R. Malacea, J.-C. Daran, S. B. Duckett, J. P. Dunne, C. Godard, E. Manoury, R. Poli, A. C. Whitwood, Dalton (2006) 3350.

[26] L. Routaboul, S. Vincendeau, C. O. Turrin, A. M. Caminade, J. P. Majoral, J. C. Daran, E. Manoury, J. Organomet. Chem. 692 (2007) 1064.

[27] E. Le Roux, R. Malacea, E. Manoury, R. Poli, L. Gonsalvi, M. Peruzzini, Adv. Synth. \& Catal. 349 (2007) 309.

[28] For reviews about ${ }^{195}$ Pt NMR, see: (a) P. S. Pregosin Coor. Chem. Rev., 44 (1982), 247. (b) J. R. L. Priqueler, I. S. Butler, F. D. Rochon, Appl. Spectr. Rev., 41 (2006), 185. (c) B. M. Still, P. G. Anil Kumar, J. R. Aldrich-Wright, W. S. Price, Chem. Soc. Rev., 36 (2007), 665.

[29] G. K. Anderson, R. Kumar, Inorg. Chem. 23 (1984) 4064.

[30] G. Parrinello, J. K. Stille, J. Am. Chem. Soc. 109 (1987) 7122.

[31] G. Petocz, Z. Berente, T. Kegl, L. Kollar, J. Organomet. Chem. 689 (2004) 1188.

[32] J. I. Van Der Vlugt, R. Van Duren, G. D. Batema, R. Den Heeten, A. Meetsma, J. Fraanje, K. Goubitz, P. C. J. Kamer, P. Van Leeuwen, D. Vogt, Organometallics 24 (2005) 5377.

[33] E. M. Al-Shami, A. S. Abu-Surrah, M. Klinga, M. Ahlgren, H. A. Hodali, Zeitschrift fuer Kristallographie - New Crystal Structures 218 (2003) 537.

[34] G. A. Molander, J. P. Burke, P. J. Carroll, J. Org. Chem. 69 (2004) 8062.

[35] D. Morales-Morales, R. Redon, Y. F. Zheng, J. R. Dilworth, Inorg. Chim. Acta 328 (2002) 39. 
[36] E. M. Al-Shami, A. S. Abu-Surrah, M. Klinga, H. A. Hodali, Z. Anorg. Allg. Chem. 628 (2002) 1433.

[37] H. Nakano, Y. Suzuki, C. Kabuto, R. Fujita, H. Hongo, J. Org. Chem. 67 (2002) 5011

[38] S. D. Toto, M. M. Olmstead, B. W. Arbuckle, P. K. Bharadwaj, W. K. Musker, Inorg. Chem. 29 (1990) 691.

[39] O. G. Mancheno, R. G. Arrayas, J. C. Carretero, Organometallics 24 (2005) 557.

[40] J. Yang, D. J. Huang, G. N. Li, L. F. Zhang, Acta Chimica Sinica 51 (1993) 1145.

[41] J. Cirera, P. Alemany, S. Alvarez, Chem. Eur. J. 10 (2004) 190.

[42] A. Altomare, M. Burla, M. Camalli, G. Cascarano, C. Giacovazzo, A. Guagliardi, A. Moliterni, G. Polidori, R. Spagna, J. Appl. Cryst. 32 (1999) 115.

[43] G. M. Sheldrick, SHELXL97. Program for Crystal Structure refinement, University of Göttingen, Göttingen, Germany, 1997.

[44] H. D. Flack, Acta Cryst. A39 (1983) 876.

[45] L. J. Farrugia, J. Appl. Crystallogr. 32 (1997) 565. 
Table 3. Crystal data and structure refinement for all compounds.

\begin{tabular}{|c|c|c|c|c|c|c|c|c|}
\hline Identification code & $2 \mathbf{a}$ & $2 \mathbf{b}$ & $2 \mathbf{b}$ & $2 c$ & $3 \mathbf{a}$ & $\mathbf{3 b}$ & $3 c$ & 3d \\
\hline Empirical formula & $\mathrm{C}_{30} \mathrm{H}_{26} \mathrm{Cl}_{5} \mathrm{FePPdS}$ & $\mathrm{C}_{25} \mathrm{H}_{25} \mathrm{Cl}_{2} \mathrm{FePPdS}$ & $\mathrm{C}_{25} \mathrm{H}_{25} \mathrm{Cl}_{2} \mathrm{FePPdS}$ & $\mathrm{C}_{27} \mathrm{H}_{29} \mathrm{Cl}_{2} \mathrm{FePPdS}$ & $\mathrm{C}_{30} \mathrm{H}_{26} \mathrm{Cl}_{5} \mathrm{FePPtS}$ & $\mathrm{C}_{25} \mathrm{H}_{25} \mathrm{Cl}_{2} \mathrm{FePPtS}$ & $\mathrm{C}_{27} \mathrm{H}_{29} \mathrm{Cl}_{2} \mathrm{FePPtS}$ & $\mathrm{C}_{29} \mathrm{H}_{31} \mathrm{Cl}_{2} \mathrm{FePPtS}$ \\
\hline Formula weight & 789.04 & 621.63 & 621.63 & 649.68 & 877.73 & 710.32 & 738.37 & 764.41 \\
\hline Temperature, $\mathrm{K}$ & $180(2) \mathrm{K}$ & $180(2)$ & $180(2)$ & $180(2)$ & $180(2)$ & $180(2)$ & $180(2)$ & $180(2)$ \\
\hline Wavelength, $\AA$ & $0.71073 \AA$ & 0.71073 & 0.71073 & 0.71073 & 0.71073 & 0.71073 & 0.71073 & 0.71073 \\
\hline Crystal system & Monoclinic & Monoclinic & Triclinic & Monoclinic & Monoclinic & Monoclinic & Monoclinic & Monoclinic \\
\hline Space group & $\mathrm{P} 2{ }_{1} / \mathrm{c}$ & $\mathrm{Cc}$ & P 1 & $\mathrm{P} 2{ }_{1} / \mathrm{c}$ & $\mathrm{P} 2{ }_{1} / \mathrm{c}$ & $\mathrm{C} \mathrm{c}$ & $\mathrm{P} 2{ }_{1} / \mathrm{n}$ & $\mathrm{P} 2{ }_{1} / \mathrm{c}$ \\
\hline $\mathrm{a}, \AA$ & $9.5438(10)$ & $8.7773(12)$ & $8.7773(12)$ & $14.9597(11)$ & $9.8065(8)$ & $8.7912(6)$ & $16.6696(12)$ & $16.9666(16)$ \\
\hline $\mathrm{b}, \AA$ & $33.331(3)$ & $23.879(4)$ & $11.6335(16)$ & $7.9180(6)$ & $28.039(3)$ & $24.0504(15)$ & $9.3976(9)$ & $7.9977(5)$ \\
\hline$\alpha,{ }^{\circ}$ & 90.0 & 90.0 & $86.165(11)$ & 90.0 & 90.0 & 90.0 & 90.0 & 90.0 \\
\hline$\beta,^{\circ}$ & $110.756(11)$ & $100.872(12)$ & $69.805(13)$ & $96.274(6)$ & $109.565(10)$ & $100.631(6)$ & $114.504(7)$ & $112.454(11)$ \\
\hline$\gamma,{ }^{\circ}$ & 90.0 & 90.0 & $79.128(12)$ & 90.0 & 90.0 & 90.0 & 90.0 & 90.0 \\
\hline $\mathrm{V}, \AA^{3}$ & $3108.7(5)$ & $2394.5(6)$ & $1197.2(3)$ & $2541.0(3)$ & $3117.4(5)$ & $2415.9(3)$ & $2539.1(3)$ & $2733.1(4)$ \\
\hline $\mathrm{Z}$ & 4 & 4 & 2 & 4 & 4 & 4 & 4 & 4 \\
\hline $\mathrm{Dx}, \mathrm{Mg} / \mathrm{m}^{3}$ & 1.686 & 1.724 & 1.724 & 1.698 & 1.870 & 1.953 & 1.932 & 1.858 \\
\hline$\mu, \mathrm{mm}^{-1}$ & 1.614 & 1.746 & 1.746 & 1.649 & 5.515 & 6.770 & 6.446 & 5.992 \\
\hline $\mathrm{F}(000)$ & 1576 & 1248 & 624 & 1312 & 1704 & 1376 & 1440 & 1496 \\
\hline$\theta$ range, ${ }^{\circ}$ & $2.28-24.16$ & $3.33-28.28$ & $3.33-28.28$ & $2.74-28.22$ & $2.20-26.03$ & $2.81-28.27$ & $2.51-26.09$ & $2.74-25.98$ \\
\hline Reflctns, collected & 19490 & 12684 & 10609 & 20310 & 29524 & 9942 & 19951 & 21184 \\
\hline Reflctns, unique [R(int)] & $4868[0.0333]$ & $5837[0.0608]$ & $7575[0.0443]$ & $6300[0.0349]$ & $5804[0.0387]$ & $4870[0.0186]$ & $4744[0.0728]$ & $5307[0.0315]$ \\
\hline Completeness, $\%$, & 98.3 & 93.6 & 99.3 & 99.8 & 94.6 & 99.8 & 94.2 & 99.2 \\
\hline Absorption correction & Multiscan & Multiscan & Multiscan & Multiscan & Multiscan & Multiscan & Empirical & Multiscan \\
\hline Max., min. transm. & $0.7738,0.7275$ & $0.8028,0.6749$ & $0.8028,0.6749$ & $0.7510,0.4512$ & $0.3858,0.2107$ & $0.3041,0.2364$ & $0.8240,0.4611$ & $0.4652,0.3792$ \\
\hline Refinement & $\mathrm{F}^{2}$ & $\mathrm{~F}^{2}$ & $\mathrm{~F}^{2}$ & $\mathrm{~F}^{2}$ & $\mathrm{~F}^{2}$ & $\mathrm{~F}^{2}$ & $\mathrm{~F}^{2}$ & $\mathrm{~F}^{2}$ \\
\hline Data/restraints/param. & $4868 / 0 / 352$ & $5837 / 2 / 281$ & $7575 / 3 / 562$ & $6300 / 0 / 301$ & 5804 / 0 / 391 & $4870 / 2 / 282$ & 4744 / 0 / 301 & $5307 / 0 / 316$ \\
\hline $\mathrm{GOF}$ on $\mathrm{F}^{2}$ & 1.013 & 0.969 & 0.984 & 1.041 & 1.104 & 1.149 & 0.928 & 1.021 \\
\hline $\mathrm{R}, \mathrm{wR} 2[\mathrm{I}>2 \sigma(\mathrm{I})]$ & $0.0310,0.0762$ & $0.0468,0.1022$ & $0.0475,0.1061$ & $0.0340,0.0853$ & $0.0285,0.0717$ & $0.0183,0.0420$ & $0.0360,0.0713$ & $0.0208,0.0459$ \\
\hline R, wR2 (all data) & $0.0403,0.0797$ & $0.0640,0.1105$ & $0.0600,0.1122$ & $0.0463,0.0963$ & $0.0338,0.0825$ & $0.0199,0.0541$ & $0.0640,0.0786$ & $0.0267,0.0473$ \\
\hline Flack's parameter & & $0.02(3)$ & $0.06(4)$ & & & $-0.008(5)$ & & \\
\hline$(\mathrm{Max} / \min ) \Delta \mathrm{e}, \mathrm{e} . \AA^{-3}$ & $1.318 /-1.000$ & $1.659 /-1.677$ & $1.252 /-1.324$ & $0.928 /-0.90$ & $1.204 /-1.891$ & $1.724 /-1.230$ & $1.107 /-0.894$ & $1.169 /-0.992$ \\
\hline
\end{tabular}

